\title{
Communication
}

\section{Polar-graded multiferroic SrMnO3 thin films}

Roger Guzmán, Laura Maurel, Eric Langenberg, Andrew R.

Lupini, Pedro Algarabel, José Ángel Pardo, and Cesar Magen

Nano Lett., Just Accepted Manuscript • DOI: 10.1021/acs.nanolett.5b04455 • Publication Date (Web): 21 Mar 2016

Downloaded from http://pubs.acs.org on March 22, 2016

\section{Just Accepted}

"Just Accepted" manuscripts have been peer-reviewed and accepted for publication. They are posted online prior to technical editing, formatting for publication and author proofing. The American Chemical Society provides "Just Accepted" as a free service to the research community to expedite the dissemination of scientific material as soon as possible after acceptance. "Just Accepted" manuscripts appear in full in PDF format accompanied by an HTML abstract. "Just Accepted" manuscripts have been fully peer reviewed, but should not be considered the official version of record. They are accessible to all readers and citable by the Digital Object Identifier (DOI®). "Just Accepted" is an optional service offered to authors. Therefore, the "Just Accepted" Web site may not include all articles that will be published in the journal. After a manuscript is technically edited and formatted, it will be removed from the "Just Accepted" Web site and published as an ASAP article. Note that technical editing may introduce minor changes to the manuscript text and/or graphics which could affect content, and all legal disclaimers and ethical guidelines that apply to the journal pertain. ACS cannot be held responsible for errors or consequences arising from the use of information contained in these "Just Accepted" manuscripts. 


\title{
Polar-graded multiferroic $\mathrm{SrMnO}_{3}$ thin films
}

\author{
Roger Guzmán ${ }^{1, \dot{*},}$, Laura Maurel ${ }^{2,3}$, Eric Langenberg ${ }^{3,5}$, Andrew R. Lupini ${ }^{4}$, Pedro A. \\ Algarabel $^{3,5}$, José A. Pardo ${ }^{1,2,6}$, César Magén ${ }^{1,3,7, *}$
}

${ }^{1}$ Laboratorio de Microscopías Avanzadas (LMA), Instituto de Nanociencia de Aragón (INA), Universidad de Zaragoza, 50018 Zaragoza, Spain

${ }^{2}$ Instituto de Nanociencia de Aragón (INA), Universidad de Zaragoza, 50018 Zaragoza, Spain

${ }^{3}$ Departamento de Física de la Materia Condensada, Universidad de Zaragoza, 50009 Zaragoza, Spain

${ }^{4}$ Materials Science and Technology Division, Oak Ridge National Laboratory, Oak Ridge, Tennessee 37831, USA

${ }^{5}$ Instituto de Ciencia de Materiales de Aragón (ICMA), Universidad de Zaragoza-CSIC, 50009

Zaragoza, Spain

${ }^{6}$ Departamento de Ciencia y Tecnología de Materiales y Fluidos, Universidad de Zaragoza, 50018 Zaragoza, Spain

${ }^{7}$ Fundación ARAID, 50004 Zaragoza, Spain 


\begin{abstract}
Engineering defects and strains in oxides provides a promising route for the quest of thin film materials with coexisting ferroic orders, multiferroics, with efficient magnetoelectric coupling at room temperature. Precise control of the strain gradient would enable custom tailoring of the multiferroic properties, but presently remains challenging. Here we explore the existence of a polar-graded state in epitaxially-strained antiferromagnetic $\mathrm{SrMnO}_{3}$ thin films, whose polar nature was predicted theoretically, and recently demonstrated experimentally. By means of aberration-corrected scanning transmission electron microscopy we map the polar rotation of the ferroelectric polarization at atomic resolution, both far from and near the domain walls and find flexoelectricity resulting from vertical strain gradients. The origin of this particular strain state is a gradual distribution of oxygen vacancies across the film thickness, according to electron energy loss spectroscopy. Herein we present a chemistry-mediated route to induce polar rotations in oxygen-deficient multiferroic films, resulting in flexoelectric polar rotations and with potentially enhanced piezoelectricity.
\end{abstract}

KEYWORDS: Multiferroics, ferroelectricity, flexoelectricity, aberration-corrected STEM, domain walls. 


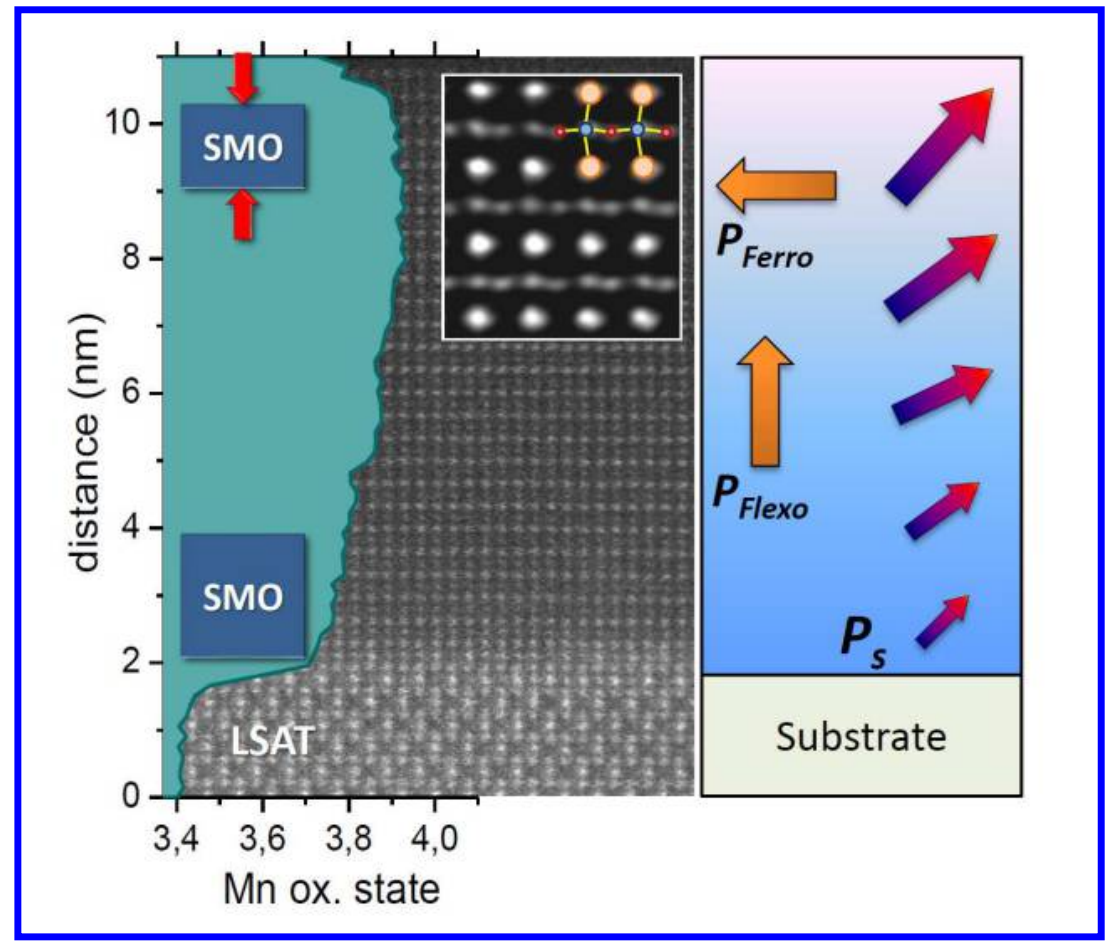

\section{Table of contents figure}


TEXT

Multiferroic materials have attracted great interest recently because of their intriguing fundamental physics and the wide range of potential applications such as transducers and information storage ${ }^{1-3}$. Of particular technological impact is the search for materials showing efficient coupling between ferromagnetic and ferroelectric orders that persist at room temperature. Manganese-based perovskite oxides $\mathrm{AMnO}_{3}, \mathrm{~A}$ being an alkaline-earth cation, are particularly promising for this purpose; theoretical calculations reveal the onset of a ferroelectric ground state with strong magnetoelectric coupling since the spontaneous polarization is expected to be driven by the off-centering of the magnetic $\mathrm{Mn}^{4+}$ ion ${ }^{4,5}$. The ferroelectric instability is predicted to increase by expansion of the lattice and, in turn, strain-induced ferroelectricity was experimentally demonstrated in Ba-substituted $\mathrm{SrMnO}_{3}$ single crystals, in which chemical expansion of the crystal lattice is caused by partially replacing $\mathrm{Sr}$ with larger $\mathrm{Ba}$ ions ${ }^{6}$.

Alternatively, strains can be induced into films through the use of epitaxial growth and can be utilized to modify the ferroelectric film properties by an adequate choice of the substrate ${ }^{7-9}$. In particular, strain-engineering of multiferroism has opened a path for the exploration of new phases with improved properties compared to their bulk parents ${ }^{10-14}$, e.g. the emergence of a ferroelectric-ferromagnetic state in $\mathrm{EuTiO}_{3}$ (ref. 14), the occurrence of incipient ferroelectricity in highly strained $\mathrm{CaMnO}_{3}$ (ref. 12), or the formation of a two-dimensional ferromagnetic phase at the domain walls of $\mathrm{TbMnO}_{3}$ (ref. 11).

Recently, the coupling between strain gradients and polarization, known as flexoelectricity ${ }^{15}$, has been analyzed intensely due to its remarkable contribution to the polarization and permittivity of inhomogeneously strained ferroelectrics ${ }^{16-21}$. The most dramatic manifestations of flexoelectricity have been reported in thin films, as it is an inherently size-dependent 
phenomenon where strain gradients are inversely proportional to size $\mathrm{e}^{19,20,22}$. Interestingly, in contrast to piezoelectricity, flexoelectricity is a universal effect due to the relaxed restriction on crystal symmetry and might emerge even in centrosymmetric crystals ${ }^{17}$, where the inversion symmetry is disrupted by the strain gradient.

Local strain gradients are common in complex materials. In dislocation-free epitaxial films, the strain is usually relieved by changing the system symmetry ${ }^{23,24}$ or through the formation of ferroelastic domains ${ }^{25}$. Large flexoelectric polarizations have been reported for dislocation-free but ferroelastically twinned films with large elastic strain gradients causing a rotation of the polarization $^{21}$; in this case, the flexoelectric polarization were comparable to the spontaneous polarization of archetypal ferroelectrics evidencing that strain-engineered polar rotations can be an alternative pathway to enhancing piezoelectricity via the generation of ferroelectrics with tailored polarization ${ }^{26,27}$.

In the present work, we focus on the precise structural analysis of the polar state in (001)oriented epitaxial $\mathrm{SrMnO}_{3}(\mathrm{SMO})$ thin films previously predicted ${ }^{13}$, and recently demonstrated ${ }^{28}$ to turn into ferroelectric state under epitaxial strain developing domains with in-plane polarization along the $\langle 110\rangle$ axes. Aberration-corrected scanning transmission electron microscopy (STEM) and the combination of annular bright-field (ABF) with high angle annular dark-field (HAADF) are used to image both light $(\mathrm{O})$ and heavy ( $\mathrm{Sr}$ and $\mathrm{Mn}$ ) elements ${ }^{29-32}$ in the polar structure and investigate, at the atomic scale level, cation-oxygen dipole distortion far from and near to the walls between domains. Local deformation analyses reveal a particular strain state where flexoelectricity, due to $\langle 001\rangle$ strain gradients, induce a gradual rotation of the inplane $\langle 110\rangle$ electric polarization. We argue that the strain-induced polar gradient is associated with the gradual distribution of point defects in the form of oxygen vacancies, opening new 
pathways for the generation of new chemistry-mediated functional multiferroic materials with custom-designed polarization orientation and enhanced piezoelectric properties.

$10 \mathrm{~nm}$ thick films of single-phase $\mathrm{SrMnO}_{3}$ were grown by pulsed laser deposition on top of (001)-oriented $\left(\mathrm{LaAlO}_{3}\right)_{0.3}\left(\mathrm{Sr}_{2} \mathrm{AlTaO}_{6}\right)_{0.7}(\mathrm{LSAT})$. LSAT substrate is used to induce a nominal in-plane epitaxial strain value of $+1.68 \%$ to the film with respect to bulk SMO. Figure 1a is a representative Z-contrast HAADF-STEM cross-sectional image of an SMO film viewed along the $\langle 110\rangle$ direction showing the high crystalline quality of the films and the absence of defects. Geometrical Phase Analysis (GPA) ${ }^{33}$, which is an efficient method to measure lattice deformation from high-resolution images (Figure 1a), evidences that the film grows fully coherent along the in-plane direction, with the same lattice parameter $\boldsymbol{a}$ as the substrate reference (Figure 1b). However, the out-of-plane deformation map, Figure 1c, reveals that the film undergoes a vertical compressive strain gradient reflecting that the film presents a tetragonal structure $(\mathrm{c} / \mathrm{a}<1)$ with increasing tetragonality towards the upper layers. Here, the out-of-plane lattice parameter $\mathrm{c}$ decreases from a deformation value of $-0.5 \pm 0.3 \%$ near the substrate interface to $-1.6 \pm 0.3 \%$ in the upper layers relative to the substrate reference $\left(\mathrm{a}_{\mathrm{LSAT}}=0.3869 \mathrm{~nm}\right)$, i.e. $\mathrm{c} \sim 0.3807 \mathrm{~nm}$, which corresponds to $+0.1 \%$ with respect to bulk SMO ( $\mathrm{a}_{\text {bulk }}=0.3805 \mathrm{~nm}$ ) (see ref. 34). The drop of the deformation values in the last unit cells near the surface is due to damage caused by the sample preparation. Notice that SMO at the interface presents a deformation of $+3.5 \pm 0.6 \%$. This tensile deformation is localized at the very first layer of the SMO film due to distributed substrate steps at the interface. This is highlighted in the inset of Figure 1a, a close up image of the interface, where the Ta columns (brightest columns) from the LSAT structure are overlapped with Mn columns in the first Mn-O plane. 

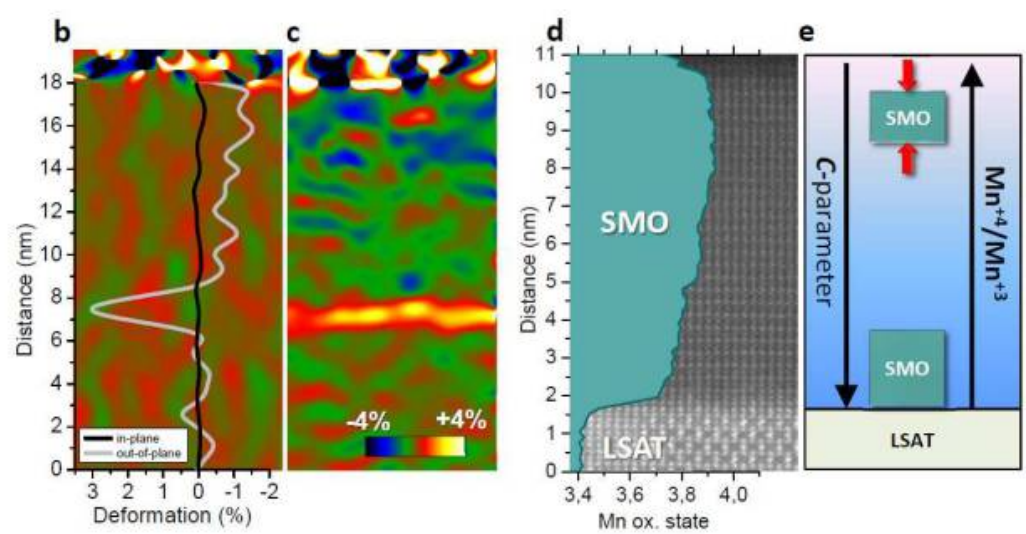

Figure 1 1 Strain state and $\mathrm{Mn}$ oxidation state of $\mathrm{SrMnO}_{3}$ thin films. a, HAADF STEM image of a cross-sectional $10 \mathrm{~nm}$ thick SMO/LSAT (001) film viewed along the $\langle 110\rangle$ direction. The inset is a close-up image of the SMO/LSAT interface where the arrow points to the overlapping of Ta and Mn columns due to the presence of substrate steps. $\mathbf{b}$ and $\mathbf{c}$ are the corresponding GPA in-plane and out-of-plane deformation maps, respectively, relative to the LSAT substrate (reference). d, Profile of the $\mathrm{SrMnO}_{3}$ film measured by EELS. The gradual increase of the oxidation state denotes a gradual distribution of oxygen vacancies. e, Sketch of the SMO film illustrating the oxygen vacancy distribution correlated with the out-of-plane lattice variation.

The presence of [001] strain gradients in the absence of dislocations or crystal twinning implies an intriguing strain-relief mechanism. Furthermore, the GPA analysis shows a particular strain state where the cell volume gradually lowers away from the interface and tetragonality increases. Generally, it is assumed that epitaxial strain is accommodated mainly by changes in the intrinsic lattice constants or structural distortions such as changing internal bond lengths or octahedral tilting in perovskites. However, for certain tensile strain values, point defect formation is also a likely strain-relaxation mechanism; it has previously been predicted that tensile strain lowers the oxygen vacancy formation energy in biaxially strained $\mathrm{CaMnO}_{3}{ }^{35}$. 
Earlier works in ferroelectric $\mathrm{HoMnO}_{3}$ epitaxial films pointed out the interplay between strain gradients and the presence of oxygen vacancies and the possibility to modulate the strain gradients, and thus flexoelectricity and polarization, as a function of the oxygen pressure during the film growth ${ }^{19}$. Here, the oxygen-pressure dependence on the perovskite phase stabilization is the direct evidence that the formation of oxygen vacancies during thin film growth plays a central role in its stability, since perovskite SMO is only stabilized under low oxygen pressure $^{28,36,37}$. The formation of oxygen vacancies is charge compensated by a reduction of the formal oxidation state of some $\mathrm{Mn}^{4+}$ to $\mathrm{Mn}^{3+}$, resulting in a chemical expansion of the crystal lattice, relieving the strain. To probe the oxygen deficiency and the distribution of the point defects in the SMO film, we used electron energy loss spectroscopy (EELS) to trace the depth profile of the local Mn oxidation state (see Supporting Information). The result is displayed in Figure 1d. The analysis of the EELS profile reveals that the Mn oxidation state increases gradually from approximately $+3.7 \pm 0.05$ to $+3.9 \pm 0.05$ towards the top of the film, indicating a gradual change in the distribution of oxygen vacancies with an increased population near the substrate interface. The drop of the oxidation state in the top is again due to film surface damage, as reflected in the GPA deformation maps. Noticeably, the higher Mn valence in the upper layers is in agreement with the decrease in volume away from interface, as sketched in Figure 1e.

To determine the precise positions of the cation and oxygen columns and their relative displacements, simultaneous HAADF and ABF images were acquired, see Figure 2a. The images qualitatively show the off-centering of both $\mathrm{Mn}$ and $\mathrm{O}$ atoms from their centrosymmetric $\operatorname{positions}^{34}$, as highlighted in Figure 2b. Figure 2c illustrates schematically the atom displacements with respect to the center of the pseudocubic perovskite cell viewed along the 
$\langle 110\rangle$ projection. Mn columns are found to shift along the in-plane (towards the [-1-10]) and outof-plane (towards the [00-1]) directions. A polar distortion with in-plane component along $\langle 110\rangle$ was already experimentally observed in biaxially strained $\mathrm{SrMnO}_{3}$ thin films ${ }^{28}$ and is in line with the traditionally accepted model of the strain-polarization coupling in most tetragonal ferroelectrics ${ }^{38}$. However, the flexoelectric component associated with the $\langle 001\rangle$ strain gradient induces a rotation of the ferroelectric distortion resulting in a [-1-1-1] displacement component. This unique polar state differs from the one observed in thicker SMO films $(20 \mathrm{~nm})$, without chemically-induced strain gradients and thus no presence of flexoelectricity (see ref. 28 and Supporting Information).

\section{a}

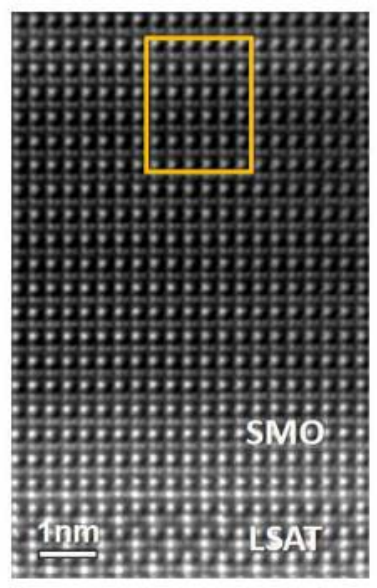

b

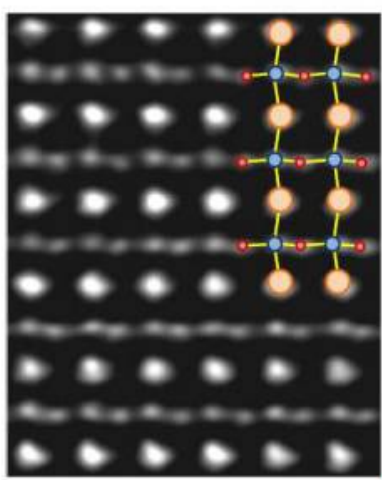

c

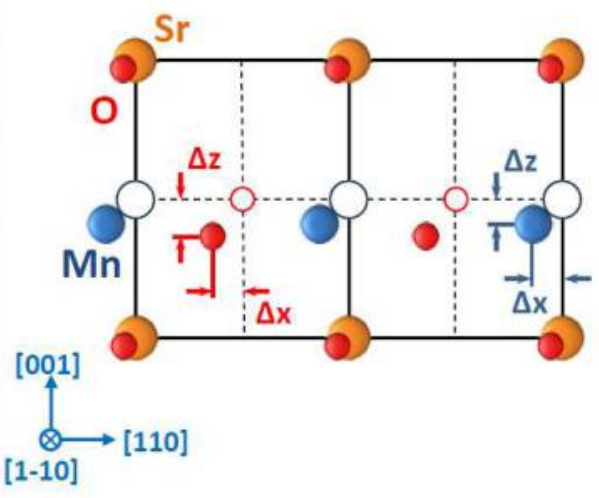

Figure 2 | Atomic-scale imaging of the polar SMO structure. a, Inverted-contrast ABF image of the SMO/LSAT interface taken along the [1-10] direction. $\mathbf{b}$, Magnification of the ABF image showing the polar SMO structure where cation and oxygen positions are indicated, Sr: orange; Mn: blue; O: red. c, Projection of the polar SMO unit cell along the [1-10] direction. $\Delta x$ and $\Delta z$ denote the shift of the $\mathrm{Mn}$ (blue) and $\mathrm{O}$ atoms (red) along the in-plane and out-of-plane direction respectively, with respect to the centrosymmetric positions (open circles). 
To analyze quantitatively the displacement of both $\mathrm{Mn}$ and $\mathrm{O}$ atoms as a function of the strain gradient we precisely measured their atomic positions from the ABF images using a center-ofmass refinement method with picometer resolution. We defined the Sr lattice as the reference to measure the relative displacements of $\mathrm{Mn}$ and $\mathrm{O}$ from centrosymmetric positions. Using this method we analyzed an area of the film comprising 41[110] by 22[001] unit cells, where a portion of the image is shown in Figure 3a. Here we define the displacement of Mn atoms (blue) and $\mathrm{O}$ atoms (red) along the in-plane direction by a distance $\boldsymbol{\Delta}_{\mathbf{x}}$, and along the out-of-plane by a distance $\boldsymbol{\Delta}_{\mathbf{z}}$ with respect to the positions of centrosymmetry (blue and red open circles) as illustrated in Figure 2c. Figure 3b shows the in-plane and the out-of-plane lattice variation by measuring the interatomic distances between A-site cations ( $\mathrm{Sr})$. In agreement with the GPA shown in Figure 1, the a-parameter remains unchanged and the c-parameter decreases gradually with increasing distance from the interface, reaching a minimum at a distance of about 20 unit cells from the interface. At this point, c reaches a value of $0.377 \mathrm{~nm}$; taking $\mathrm{a}(100)=0.387 \mathrm{~nm}$, this translates into a tetragonality value of $\mathrm{c} / \mathrm{a} \approx 0.97$. Figure $3 \mathbf{c}$ and Figure $3 \mathbf{d}$ show the values of the shift parameters $\Delta_{\mathbf{z}}$ and $\Delta_{\mathbf{x}}$, respectively, for both $\mathrm{Mn}$ and $\mathrm{O}$ atoms as a function of the unit-cell position from the interface. Interestingly, both $\boldsymbol{\Delta}_{\mathbf{z}}$ and $\boldsymbol{\Delta}_{\mathbf{x}}$ scale with the strain gradient reaching the maximum off-center displacements in the upper layers (the tetragonality maxima). 


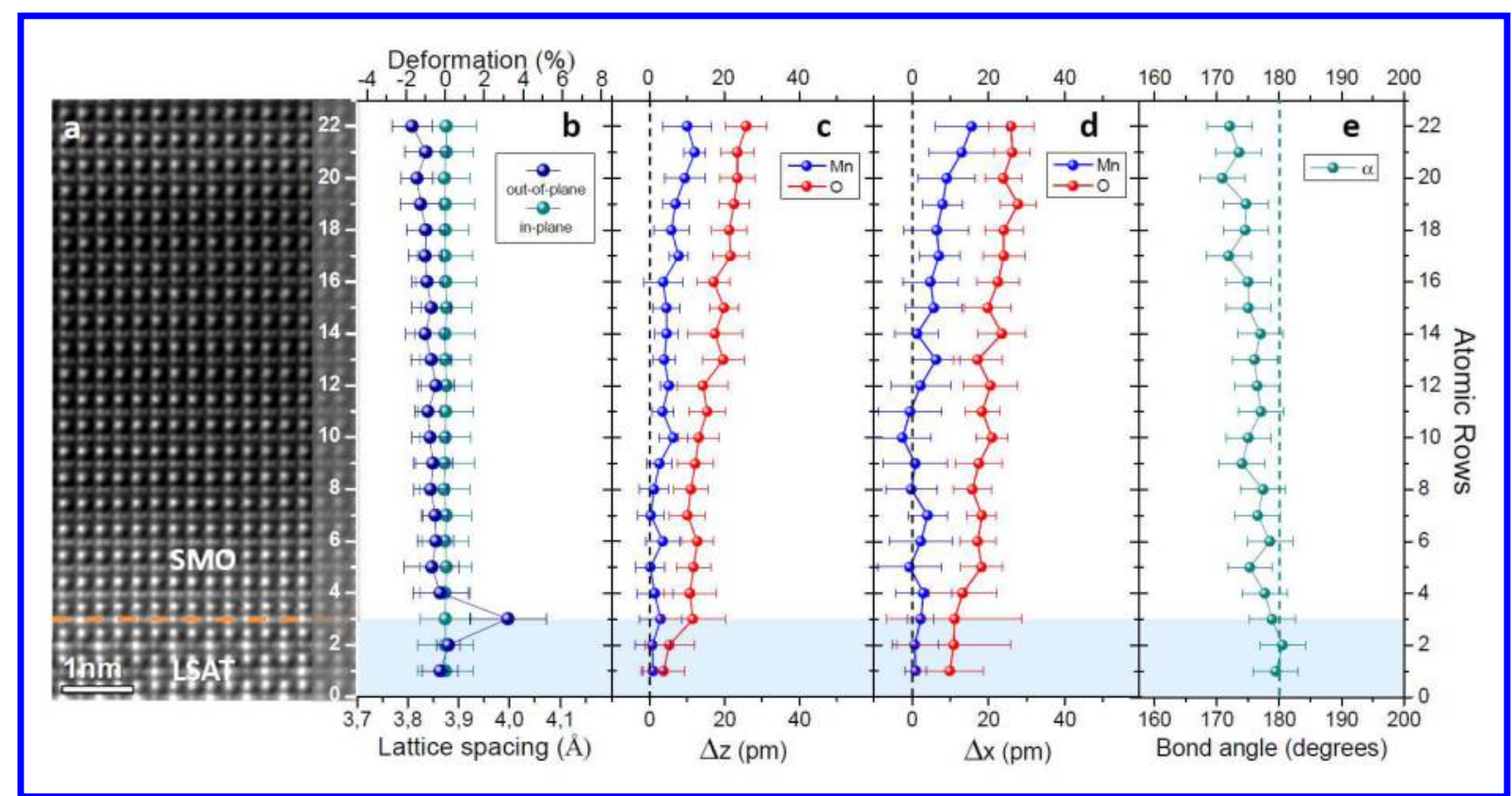

Figure 3 Quantitative analysis of the Mn and $O$ displacements and bond angles across the

SMO film. a, Portion of the ABF image shown in Figure 2. b, Variation of the out-of-plane (blue) and in-plane (green) lattice spacing. $\mathbf{c}$ and $\mathbf{d}$, Mn and O out-of-plane $\left(\boldsymbol{\Delta}_{\mathbf{z}}\right)$ and in-plane $\left(\boldsymbol{\Delta}_{\mathbf{x}}\right)$ atomic displacements extracted from the ABF image in Figure 2a, respectively. e, Variation of the Mn-O-Mn bond angle, $\alpha$, calculated from the $\boldsymbol{\Delta}_{\mathbf{z}}$ values. The interatomic distances, displacements and angles were determined by averaging over the $10_{[110]}$ unit cells per atomic row. The error bars show standard deviation with respect to averaging for each lattice layer.

Although both $\mathrm{Mn}$ and $\mathrm{O}$ atoms shift similarly they present different range values. In the case of the out-of-plane displacements $\boldsymbol{\Delta}_{\mathbf{z}}$, while Mn shifts gradually from $\sim 0 \mathrm{pm}$ near the interface to $12 \mathrm{pm}$ away from interface, $\mathrm{O}$ varies from $\sim 10$ to $25 \mathrm{pm}$. The larger $\mathrm{O}$ displacement with respect to $\mathrm{Mn}$ leads to a decrease of the Mn-O-Mn bond angle $\alpha$ (see Figure 3b) and thus $\mathrm{O}$ is no longer collinear with the Mn atoms. Following the same trend, $\alpha$ scales with $\boldsymbol{\Delta}_{\mathbf{z}}$ and decreases from $\sim 178^{\circ}$ down to $\sim 172^{\circ}$, Figure 3e. In the same way, larger $\boldsymbol{\Delta}_{\mathbf{x}}$ values for oxygen entail an asymmetric distance between the $\mathrm{Mn}$ and its nearest neighbor oxygens in the $\mathrm{MnO}_{4}$ plane, the 
Mn-O bond length. In previous low-energy muon spectroscopy experiments, performed in the same sample, we obtained a broad decay in the transverse fraction of the muons having their spin initially aligned transversal to the local magnetic field direction ${ }^{36}$. This behavior has been associated to the existence of a distribution of transition temperatures whose origin can be attributed to the gradient of the Mn-O-Mn bond angles, which produces a variation of the superexchange paths yielding local changes in the Néel temperature. The Mn-O-Mn bond angle measured here is in good agreement with the predictions on the multiferroic phase of the manganite $\mathrm{Sr}_{0.5} \mathrm{Ba}_{0.5} \mathrm{MnO}_{3}$ where $\alpha$ decreases with the $\mathrm{Mn}$ off-centering stabilizing the ferroelectric polarization ${ }^{39}$. Calculations predict an angle $\alpha \sim 175^{\circ}$ for a tetragonality value of $\mathrm{c} / \mathrm{a}=$ 0.99, which corresponds to a lattice parameter for SMO of approximately $0.384 \mathrm{~nm}$ and a deformation value of $-0.8 \%$, according to Figure $3 \mathbf{b}$.

This new atom arrangement of the polar structure leads to a separation of the center of the negative charge of oxygen anions from that of the positive charge of the cations. The displacements observed here entail a complex scenario where the vertical flexoelectricity coupled to the horizontal ferroelectric distortion should result in a rotated polarization. Besides, the apical oxygen positions of the $\mathrm{MnO}_{6}$ octahedra are seen end-on in the $\langle 110\rangle$ projection, overlapped with Sr columns, and remain undetermined. With the aim of fully resolving the SMO crystal structure and define the vector of spontaneous polarization, additional [100]-crosssectional and [001]-plane-view specimens were prepared and investigated by combined ABF and HAADF imaging (see Supporting Information). The observation of the Mn and O lattice along $\langle 110\rangle,\langle 100\rangle$ and $\langle 001\rangle$ projections confirmed that both oxygen and manganese lattices shift in the same direction. This should give a linear relation between the spontaneous polarization $\mathbf{P}_{\mathbf{s}}$ and the displacement of $\mathrm{Mn}$ atoms with respect to the oxygen octahedron, as it is well known for 
tetragonally-distorted ferroelectrics ${ }^{40-42}$. Therefore we can estimate the flexoelectricity and its effect in the modulus and angle of the spontaneous polarization. Figure $4 \mathbf{a}$ shows $\mathbf{P}_{\mathbf{x}}$ (ferro) and $\mathbf{P}_{\mathbf{z}}$ (flexo) polarization components and modulus and angle of $\mathbf{P}_{\mathbf{s}}$ as a function of the distance from the interface calculated on the basis of the determined atomic displacements shown in Figure 3 and the born effective charge (BCP) values of ions for the strained isoelectronic $\mathrm{CaMnO}_{3}$ given in ref. 4. As shown, while $\mathbf{P}_{\mathbf{z}}$ scales from 30 to $55 \mu \mathrm{C} / \mathrm{cm}^{2}$, the maximum value of the modulus of $\mathbf{P}_{\mathbf{x}}$ is about $65 \mu \mathrm{C} / \mathrm{cm}^{2}$ in the center of the film. The large vertical flexoelectric polarization will cause the spontaneous polarization vector to rotate, as shown schematically in Figure 4b; taking an average $\mathbf{P}_{\mathbf{z}} \sim 45 \mu \mathrm{C} / \mathrm{cm}^{2}$ and $\mathbf{P}_{\mathbf{x}}=35-65 \mu \mathrm{C} / \mathrm{cm}^{2}$ this results in a polar rotation angle of $30^{\circ}-50^{\circ}$ (Figure 4a). Furthermore, knowing the strain state from the GPA analysis (Figure 1), the flexoelectric coefficient can be also determined. Given an out-of-plane strain difference $\Delta \varepsilon \sim 0.01$ between the interface and the maximum deformation value over a relaxation length $t \sim 8 \mathrm{~nm}$, a strain gradient of $1.25 \times 10^{6} \mathrm{~m}^{-1}$ is obtained, which is 6 times larger than the values reported for bulk solids (typically in the order of $0.1 \mathrm{~m}^{-1}$ ) and an order of magnitude larger than the gradients reported for $\mathrm{HoMnO}_{3}$ epitaxial thin films with giant flexoelectricity ${ }^{19}$. Then, the longitudinal flexoelectric coefficient should be about $\mu=\mathbf{P}_{\mathbf{z}(\mathbf{f l e x o})} /(\Delta \varepsilon / t) \approx 400 \mathrm{nC} / \mathrm{m}$. Notice that this number is much higher than the few $\mathrm{nC} / \mathrm{m}$ measured in $\mathrm{SrTiO}_{3}$ single crystals and ceramics ${ }^{17,43}$, an order of magnitude lower than that of the few $\mu \mathrm{C} / \mathrm{m}$ measured in bulk lead-based ferroelectrics ${ }^{16}$ and far from the several $\mu \mathrm{C} / \mathrm{m}$ in $\mathrm{BaSrTiO}_{3}$ ceramics ${ }^{44}$. Nevertheless, both flexoelectric coefficient and polarization are estimated from the BECs given for another compound and real calculations out of a strain-graded oxygendeficient SMO system are needed to provide more reliable values. 


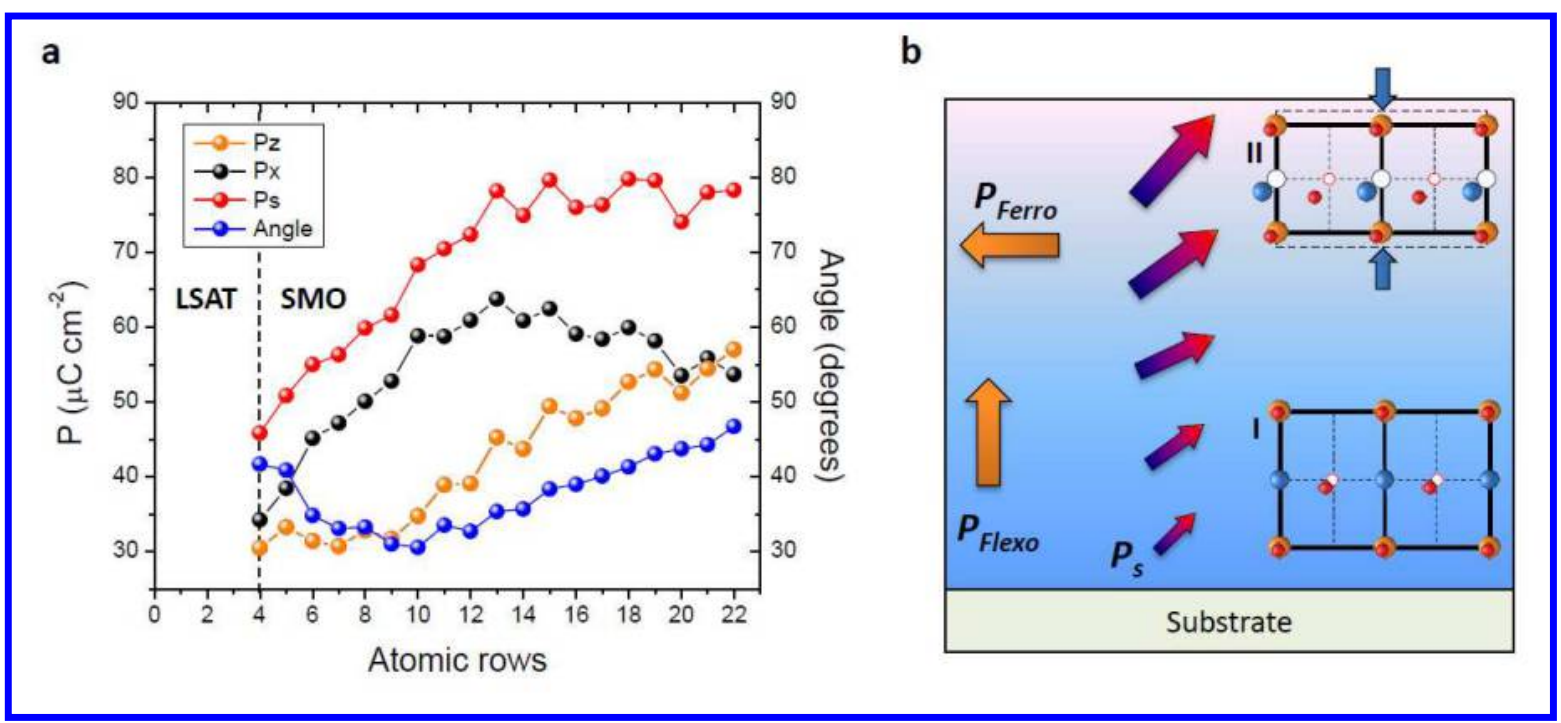

Figure 4 | Polarization and polar rotation in the SMO structure. $\mathbf{a}, \mathbf{P}_{\mathbf{x}}$ (ferro) and $\mathbf{P}_{\mathbf{z}}$ (flexo) polarization components and modulus and angle of $\mathbf{P}_{\mathbf{s}}$ as a function of the distance from the interface. b, Schematic representation of the polar graded SMO film. The fleoxelectric polarization $\left(P_{\text {flexo }}\right)$ induces a rotation of the ferroelectric polarization $\left(P_{\text {ferro }}\right)$ as represented by the coloured arrows, spontaneous polarization $\left(P_{s}\right)$. The size and angle of the arrows denote the magnitude and angle of the $\mathbf{P}_{\mathbf{s}}$, respectively, as function of the strain gradient and ferroelectric distortion. I and II are schematic representations of the polar SMO unit cell with increased tetragonality towards the upper layers. Cation and oxygen positions are indicated, Sr: orange; Mn: blue; O: red. Open circles represent the centrosymmetric positions.

The microstructure of the domain walls was carefully examined by an accurate displacement mapping of extended regions of the film. Domains of opposite and perpendicular polarization (see Supporting Information) were observed with average domain sizes between 100 and 400 $\mathrm{nm}$, which is in line with previous observations of the domain structure ${ }^{28}$. Figure 5a shows an $18 \mathrm{~nm}$-width ABF image of the SMO film, where atomic shifts were calculated within the 
squared area. The resulting displacement maps are displayed in Figure 5b. On the basis of the displacement maps, the polarization components $\mathbf{P}_{\mathbf{x}(\mathbf{f e r r o})}$ and $\mathbf{P}_{\mathbf{z}(\mathrm{flexo})}$, and the modulus and angle of $\mathbf{P}_{\mathbf{s}}$, Figure 5c, were again calculated using the same procedure described in Figure 4. Here each pixel in the maps represents the displacement, polarization and vector angle values, respectively, for each atomic row, as given in the color scale bar. Interestingly, while $\mathbf{P}_{\mathbf{z}(f l e x o)}$ appears constant along the in-plane direction, the $\mathbf{P}_{\mathbf{x}(\mathbf{f e r r o})}$ map shows two domains with different polarization values. $\boldsymbol{\Delta}_{\mathbf{x}}$ maps evidence that the atoms shift gradually from one side of the image to the other. Figure 5d and Figure 5e are profiles of the Mn- $\boldsymbol{\Delta}_{\mathbf{x}} / \mathbf{O}-\boldsymbol{\Delta}_{\mathbf{x}}$ and $\mathbf{M n}-\boldsymbol{\Delta}_{\mathbf{z}} / \mathbf{O}-\boldsymbol{\Delta}_{\mathbf{z}}$ maps taken in the upper part of the pixel maps integrating 10 [001] unit cells, respectively. As shown, the Mn polarizes gradually to the opposite direction over an extended $\sim 10 \mathrm{~nm}$-thick oblique domain wall ( $\sim 18$ unit cells), and the minimum Mn displacement value occurs in the domainwall center, as shown in the ABF images in Figure 5f. The Mn inversion is accompanied by a shift of the oxygen to values close to its centrosymmetric position. As shown in the polarization maps, the right hand side domain presents a decreased polarization being the maximum value of $\mathbf{P}_{\mathbf{x}}$ and $\mathbf{P}_{\mathbf{s}}$ up to 40 and $60 \mu \mathrm{C} / \mathrm{cm}^{2}$, respectively. This reduced polarization is strictly correlated to its different polar structure since the polar distortion appears to be mainly driven by the $\mathrm{Mn}$ displacement. Although these low-polar state domains are frequently seen in these samples, its nature still remains unknown. The corresponding charge dipoles will define the direction of the vector of spontaneous polarization. Since $\mathbf{P}_{\mathbf{z}}$ is constant along the in-plane direction and $\mathbf{P}_{\mathbf{x}}$ decreases, the larger flexoelectric polarization induces a rotation of the in-plane polarization of about $\sim 15^{\circ}$, as shown in the angle map in Figure 5c and sketched in Figure 5f.

The presence of extended domain walls in ferroelectric materials has been a matter of intense debate. Recent works reported domain-wall thickness differences between charged an uncharged 
ferroelectric- $180^{\circ}$ and ferroelastic- $90^{\circ}$ walls quantitatively measured at the atomic scale in epitaxial $\mathrm{PbTiO}_{3}$ thin films ${ }^{41,42}$, in which the strong depolarizing field of the head-to-head dipoles is stabilized by distributing the polarization charge over an extended thickness ${ }^{41,45}$. However, in multiferroic materials domain walls are generally thicker than in normal ferroelectrics which may depend on the degree of coupling and correlation length between the two ferroic orders ${ }^{11,46-}$ 48. Herein, moreover, high-polar and low-polar state domains can interact together with the presence of in-plane and out-of-plane polarization gradients. In order for this configuration to be stable, the polarization discontinuities may be screened by the presence of charged defects, such as the ubiquitous oxygen vacancies in such films localized at domain walls ${ }^{28}$, accommodating the locally different symmetry.

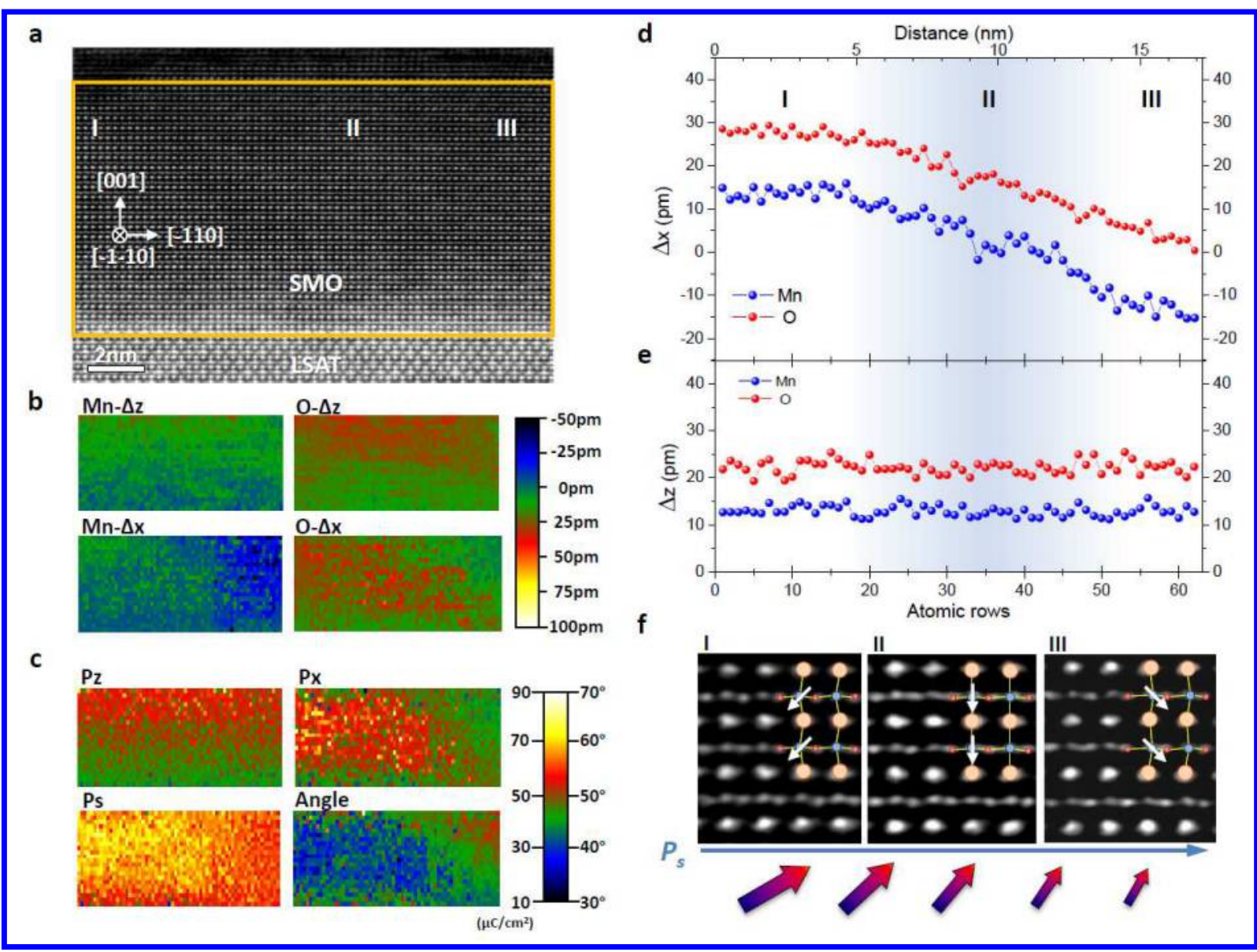




\title{
Figure 5 | Atomic level mapping of the Mn and $O$ displacement and polarization near to the
}

\author{
domain walls. a, ABF image of a larger segment of the SMO/LSAT interface. $\mathbf{b}$, Mn and O
} displacement maps for the out-of-plane $\left(\boldsymbol{\Delta}_{\mathbf{z}}\right)$ and in-plane $\left(\boldsymbol{\Delta}_{\mathbf{x}}\right)$ direction calculated from the squared region in $a$. c, $\mathbf{P}_{\mathbf{x}(\text { ferro) }}$ and $\mathbf{P}_{\mathbf{z}(\text { flexo) }}$ polarization components (in $\mu \mathrm{C} / \mathrm{cm}^{2}$ ) and modulus (in $\mu \mathrm{C} / \mathrm{cm}^{2}$ ) and angle (in degrees) of $\mathbf{P}_{\mathbf{s}}$ calculated on the basis of the displacement maps given in $b$. d, $\boldsymbol{\Delta}_{\mathbf{x}}$ and $\mathbf{e}, \boldsymbol{\Delta}_{\mathbf{z}}$ displacement profiles of the $\mathrm{Mn}$ and $\mathrm{O}$ taken from the upper part of the corresponding maps. Displacements were determined by averaging over the 10 unit cells for each atomic row. The shadowed region in the profiles depicts the thickness of the domain wall $(\sim 10$ nm) f, Magnifications of the dipoles taken from the labelled regions I, II and III in $a$ and $d$. Dipoles are formed by the displacements of ions in the unit cells, Sr: orange; Mn: blue; O: red. White arrows point the direction of displacement of the Mn atoms while the coloured arrows, labelled as $P_{s}$, show the direction and angle of the vector of spontaneous polarization.

To conclude, our findings show a chemistry-mediated route to induce polar rotations in polargraded SMO multiferroic thin films. Strain-gradient engineering through the formation of oxygen vacancies generates a $\langle 001\rangle$ flexoelectric component that rotates the in-plane $\langle 110\rangle$ ferroelectric polarization. Since polar rotations are thought to explain the giant piezoelectric response in morphotrophic phase boundary ferroelectrics ${ }^{26,27}$, this mechanism may provide an alternative strategy to design materials with high piezoelectric efficiency in multiferroic materials. Moreover, the observed thickness dependence on the polar gradient evidences that film thickness may tailor oxygen vacancies distribution and thus strain. Furthermore, regarding multiferroicity, the interplay between flexoelectricity and another order parameter coupling, for 
instance the flexomagnetoelectric effect ${ }^{49,50}$ (strain-gradient induced magnetization), may stimulate more comprehensive studies in the area of antiferromagnetic multiferroics.

\title{
ASSOCIATED CONTENT
}

Supporting Information. Details on film growth and STEM, together with supplementary images and EELS analysis on [110] cross-sectional thin films, [100]-cross sectional $10 \mathrm{~nm}$ thin films and [001] plane-view specimens are given. This material is available free of charge via the Internet at http://pubs.acs.org.

\section{AUTHOR INFORMATION}

\section{Corresponding Author}

*Email: roger.guzman.aluja@gmail.com, cmagend@unizar.es

\section{Present Addresses}

† Departamento de Física Aplicada III, Universidad Complutense de Madrid, Madrid 28040, Spain

\author{
Author Contributions \\ R.G. conducted the STEM experiments, specimen preparation and STEM data analysis. L.M. \\ grew the films. L.M. and E.L. carried out the macroscopic characterization. A.R.L performed \\ image analysis and scripting. P.A.A., J.A.P. and C.M. supervised the different activities. C.M. \\ coordinated the project. All the authors discussed the results and contributed to the final \\ manuscript.
}




\section{Notes}

The authors declare no competing financial interests.

\section{ACKNOWLEDGMENT}

Financial support from Spanish Ministerio de Economía y Competitividad through the project MAT2014-51982-C2 and from regional Gobierno de Aragón through project E26 with European Social Fund funding is acknowledged. R.G. and C.M. were funded by the European Union under the Seventh Framework Programme under a contract for an Integrated Infrastructure Initiative Reference 312483-ESTEEM2. R.G. was also supported by the ERC StG "STEMOX” 239739. A.R.L. acknowledges support by the Materials Sciences and Engineering Division, Basic Energy Sciences, Office of Science, U.S. Department of Energy (ARL) 


\section{REFERENCES}

(1) Spaldin, N. A.; Fiebig, M. Science 2005, 309 (5733), 391-392.

(2) Ramesh, R.; Spaldin, N. A. Nat. Mater. 2007, 6 (1), 21-29.

(3) Cheong, S.-W.; Mostovoy, M. Nat.Mater. 2007, 6, 13-20.

(4) Bhattacharjee, S.; Bousquet, E.; Ghosez, P. Phvs.Rev._Lett. 2009, 102 (March), 117602.

(5) Rondinelli, J. M.; Eidelson, A. S.; Spaldin, N. A. Phvs Rev. B - Condens Matter Mater. Phvs. 2009, 79 (April), 205119.

(6) Sakai, H.; Fujioka, J.; Fukuda, T.; Okuyama, D.; Hashizume, D.; Kagawa, F.; Nakao, H.; Murakami, Y.; Arima, T.; Baron, a. Q. R.; Taguchi, Y.; Tokura, Y. Phvs.Rev.Lett. 2011, 107 (13), 137601.

(7) Schlom, D. G.; Chen, L.-Q.; Eom, C.; Rabe, K. M.; Streiffer, S. K.; Triscone, J. Annu. Rev. Mater. Res. 2007, 37 (1), 589-626.

(8) Haeni, J. H.; Irvin, P.; Chang, W.; Uecker, R.; Reiche, P.; Li, Y. L.; Choudhury, S.; Tian, W.; Hawley, M. E.; Craigo, B.; Tagantsev, a K.; Pan, X. Q.; Streiffer, S. K.; Chen, L. Q.; Kirchoefer, S. W.; Levy, J.; Schlom, D. G. Nature 2004, 430 (7001), 758-761.

(9) Choi, K. J.; Biegalski, M.; Li, Y. L.; Sharan, A.; Schubert, J.; Uecker, R.; Reiche, P.; Chen, Y. B.; Pan, X. Q.; Gopalan, V.; Chen, L.-Q.; Schlom, D. G.; Eom, C. B. Science 2004, 306 (5698), 1005-1009.

(10) Wang, J.; Neaton, J. B.; Zheng, H.; Nagarajan, V.; Ogale, S. B.; Liu, B.; Viehland, D.; Vaithyanathan, V.; Schlom, D. G.; Waghmare, U. V; Spaldin, N. a; Rabe, K. M.; Wuttig, M.; Ramesh, R. Science 2003, 299 (5613), 1719-1722.

(11) Farokhipoor, S.; Magén, C.; Venkatesan, S.; Íñiguez, J.; Daumont, C. J. M.; Rubi, D.; Snoeck, E.; Mostovoy, M.; de Graaf, C.; Müller, A.; Döblinger, M.; Scheu, C.; Noheda, B. Nature 2014, 515 (7527), 379-383.

(12) Günter, T.; Bousquet, E.; David, A.; Boullay, P.; Ghosez, P.; Prellier, W.; Fiebig, M. Phvs. Rev. B - Condens. Matter Mater. Phvs. 2012, 85 (21), 214120.

(13) Lee, J. H.; Rabe, K. M. Phvs.Rev.Lett. 2010, 104 (20), 207204.

(14) Lee, J. H.; Fang, L.; Vlahos, E.; Ke, X.; Jung, Y. W.; Kourkoutis, L. F.; Kim, J.-W.; Ryan, P. J.; Heeg, T.; Roeckerath, M.; Goian, V.; Bernhagen, M.; Uecker, R.; Hammel, P. C.; Rabe, K. M.; Kamba, S.; Schubert, J.; Freeland, J. W.; Muller, D. A.; Fennie, C. J.; Schiffer, P.; Gopalan, V.; Johnston-Halperin, E.; Schlom, D. G. Nature 2010, 466 (7309), 954-958.

(15) Kogan, S. M. Sov. Phys. Solid State 1964, 5 (10), 2069-2070.

(16) Ma, W. Phvs status solidi 2008, 245 (4), 761-768.

(17) Zubko, P.; Catalan, G.; Buckley, A.; Welche, P. R. L.; Scott, J. F. Phvs.Rev._Lett. 2007, 99 (16), 167601.

(18) Zubko, P.; Catalan, G.; Tagantsev, A. K. Annu.Rev.Mater Res. 2013, 43 (1), 387-421. 
(19) Lee, D.; Yoon, A.; Jang, S. Y.; Yoon, J. G.; Chung, J. S.; Kim, M.; Scott, J. F.; Noh, T. W. Phvs. Rev.Lett. 2011, 107 (5), 057602.

(20) Catalan, G.; Sinnamon, L. J.; Gregg, J. M. L.Phvs. Condens Matter 2004, 16 (13), 22532264.

(21) Catalan, G.; Lubk, a.; Vlooswijk, a. H. G.; Snoeck, E.; Magen, C.; Janssens, a.; Rispens, G.; Rijnders, G.; Blank, D. H. a.; Noheda, B. Nat.Mater. 2011, 10 (12), 963-967.

(22) Majdoub, M. S.; Sharma, P.; Çağin, T. Phvs. Rev. B - Condens. Matter Mater. Phvs. 2008, 78 (12), 121407.

(23) Pertsev, N. A.; Zembilgotov, A. G.; Tagantsev, A. K. Phvs. Rev. Lett. 1998, 80 (9), $1988-$ 1991.

(24) Catalan, G.; Janssens, A.; Rispens, G.; Csiszar, S.; Seeck, O.; Rijnders, G.; Blank, D. H. a; Noheda, B. Phvs. Rev.Lett. 2006, 96 (12), 127602.

(25) Pompe, W.; Gong, X.; Suo, Z.; Speck, J. S. J.Appl.Phvs. 1993, 74 (10), 6012-6019.

(26) Fu, H.; Cohen, R. Nature 2000, 403 (6767), 281-283.

(27) Guo, R.; Cross, L. E.; Park, S.-E.; Noheda, B.; Cox, D. E.; Shirane, G. Phvs. Rev.Lett. 2000, 84 (23), 5423-5426.

(28) Becher, C.; Maurel, L.; Aschauer, U.; Lilienblum, M.; Magén, C.; Meier, D.; Langenberg, E.; Trassin, M.; Blasco, J.; Krug, I. P.; Algarabel, P. A.; Spaldin, N. A.; Pardo, J. A.; Fiebig, M. Nat. Nanotechnol. 2015, 10 (June), 661-665.

(29) Findlay, S. D.; Shibata, N.; Sawada, H.; Okunishi, E.; Kondo, Y.; Ikuhara, Y. Ultramicroscopv 2010, 110 (7), 903-923.

(30) Findlay, S. D.; Shibata, N.; Sawada, H.; Okunishi, E.; Kondo, Y.; Yamamoto, T.; Ikuhara, Y. Appl.Phvs.Lett. 2009, 95 (19), 191913.

(31) Haruta, M.; Kurata, H. Sci.Rep. 2012, 2, 252.

(32) Pennycook, S. J.; Jesson, D. E. Ultramicroscopy 1991, 37 (1), 14-38.

(33) Hÿtch, M. J.; Snoeck, E.; Kilaas, R. Ultramicroscopv 1998, 74 (3), 131-146.

(34) Chmaissem, O.; Dabrowski, B.; Kolesnik, S.; Mais, J.; Brown, D.; Kruk, R.; Prior, P.; Pyles, B.; Jorgensen, J. Phvs_Rev.B 2001, 64 (13), 134412.

(35) Aschauer, U.; Pfenninger, R.; Selbach, S. M.; Grande, T.; Spaldin, N. A. Phvs. Rev. B Condens. Matter Mater. Phvs. 2013, 88, 054111.

(36) Maurel, L.; Marcano, N.; Prokscha, T.; Langenberg, E.; Blasco, J.; Guzmán, R.; Suter, A.; Magén, C.; Morellón, L.; Ibarra, M. R.; Pardo, J. a.; Algarabel, P. a. Phvs.Rev. B 2015, 92 (2), 024419.

(37) Langenberg, E.; Guzmán, R.; Maurel, L.; Martínez de Baños, L.; Morellón, L.; Ibarra, M. R.; Herrero-Martín, J.; Blasco, J.; Magén, C.; Algarabel, P. A.; Pardo, J. A. ACS Annl. Mater. Interfaces 2015, 7 (43), 23967-23977.

(38) Cohen, R. E. Nature 1992, 358 (6382), 136-138. 
(39) Giovannetti, G.; Kumar, S.; Ortix, C.; Capone, M.; van den Brink, J. Phvs. Rev.Lett. 2012, 109 (10), 107601.

(40) Jia, C.-L.; Nagarajan, V.; He, J.-Q.; Houben, L.; Zhao, T.; Ramesh, R.; Urban, K.; Waser, R. Nat. Mater. 2007, 6 (1), 64-69.

(41) Jia, C.-L.; Mi, S.-B.; Urban, K.; Vrejoiu, I.; Alexe, M.; Hesse, D. Nat.Mater. 2008, 7 (1), 57-61.

(42) Tang, Y. L.; Zhu, Y. L.; Wang, Y. J.; Wang, W. Y.; Xu, Y. B.; Ren, W. J.; Zhang, Z. D.; Ma, X. L. Sci.Ren. 2014, 4, 4115.

(43) Kholkin, A.; Bdikin, I.; Ostapchuk, T.; Petzelt, J. Appl. Phvs. Lett. 2008, 93 (22), 20062009.

(44) Ma, W.; Cross, L. E. Appl. Phvs. Lett. 2006, 88 (23), 2004-2007.

(45) Eliseev, E. A.; Morozovska, A. N.; Svechnikov, G. S.; Gopalan, V.; Shur, V. Y. Phvs. Rev. B - Condens. Matter Mater. Phvs. 2011, 83 (23), 235313.

(46) Fiebig, M.; Goltsev, A. .; Lottermoser, T.; Pisarev, R. . L.Magn. Magn. Mater. 2004, 272$276,353-354$.

(47) Catalan, G.; Béa, H.; Fusil, S.; Bibes, M.; Paruch, P.; Barthélémy, a.; Scott, J. F. Phvs. Rev.Lett. 2008, 100 (2), 35-38.

(48) Daraktchiev, M.; Catalan, G.; Scott, J. F. Phvs.Rev.B 2010, 81 (22), 224118.

(49) Lukashev, P.; Sabirianov, R. F. Phvs. Rev. B - Condens. Matter Mater. Phvs. 2010, 82 (9), 094417.

(50) Pyatakov, A. P.; Zvezdin, A. K. Eur.Phvs.J.B 2009, 71 (3), 419-427. 

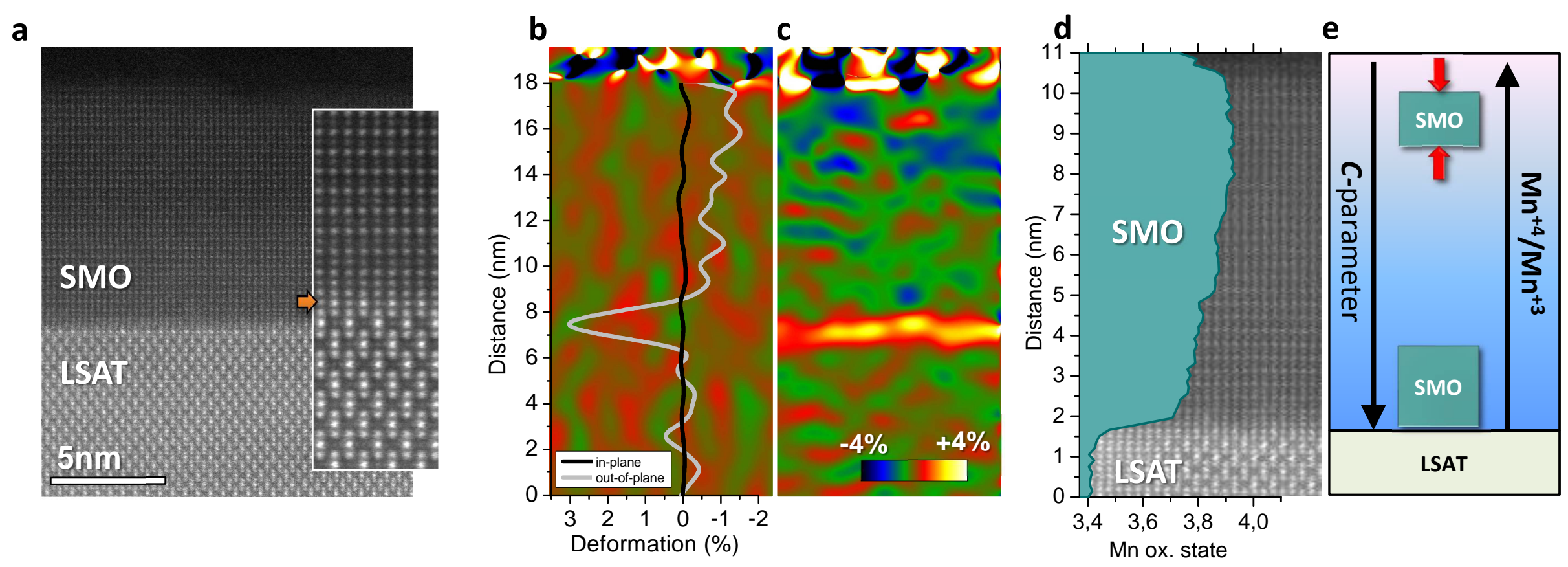
a

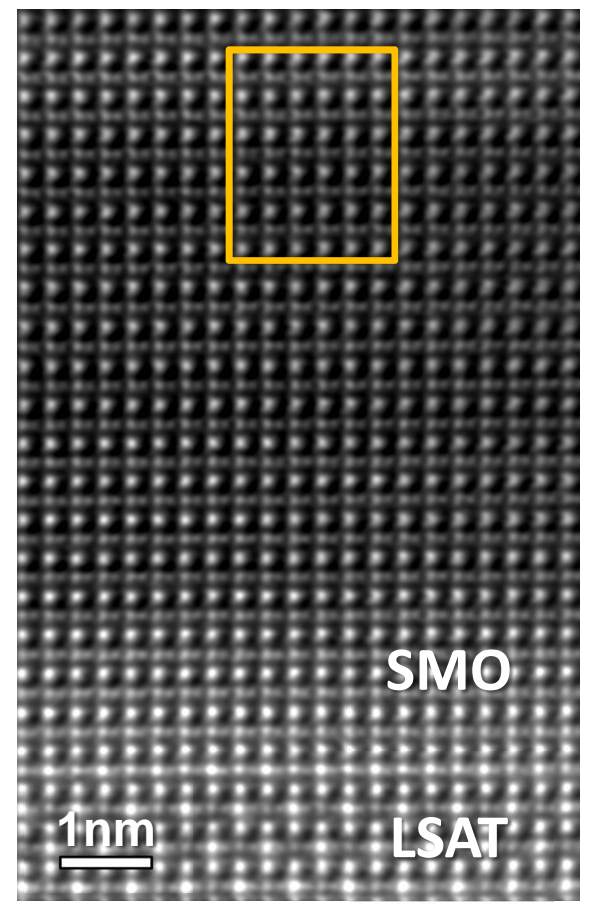

b

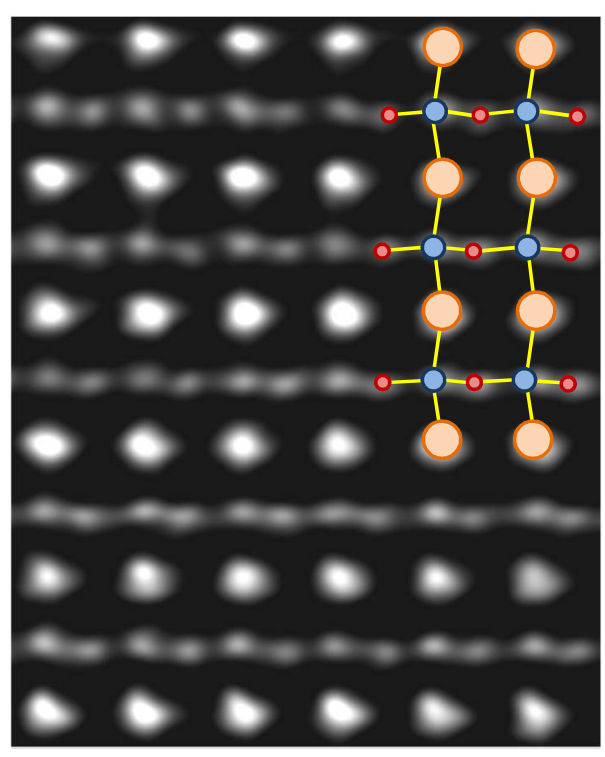

C

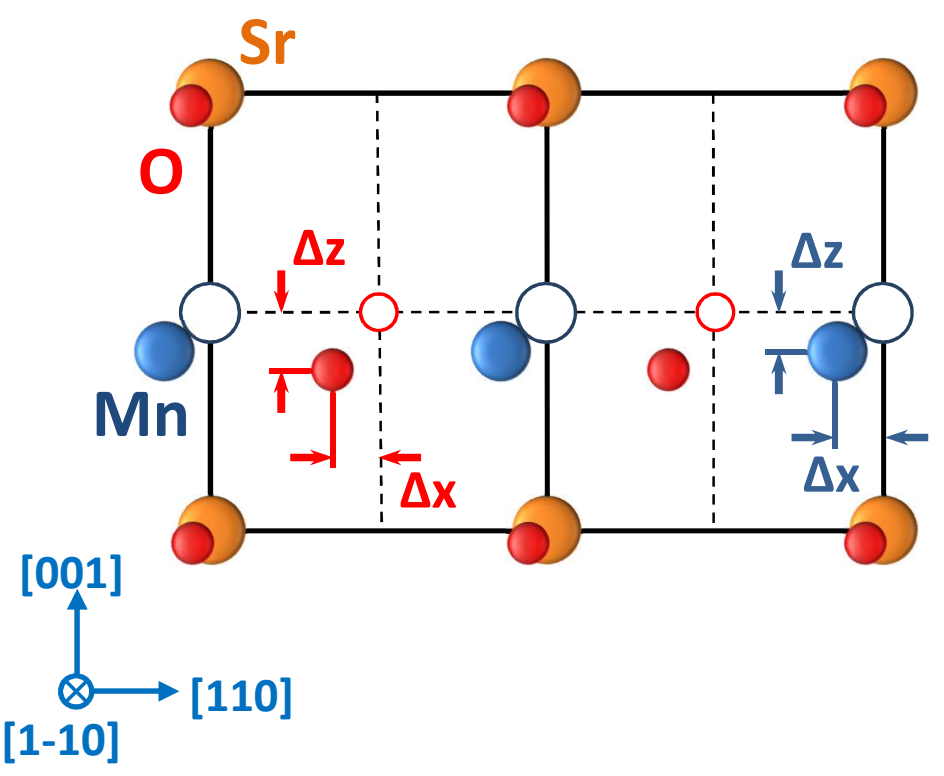




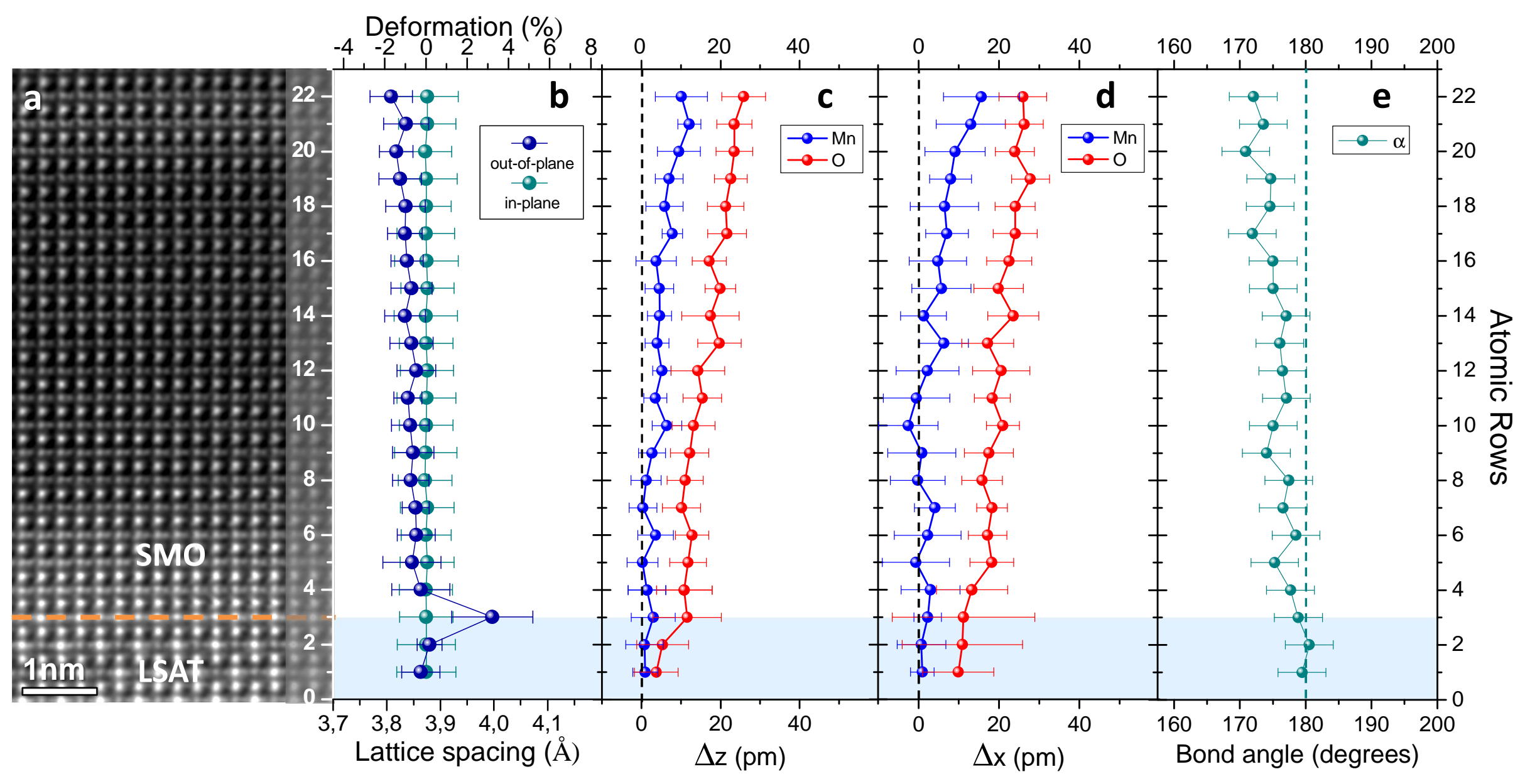


a

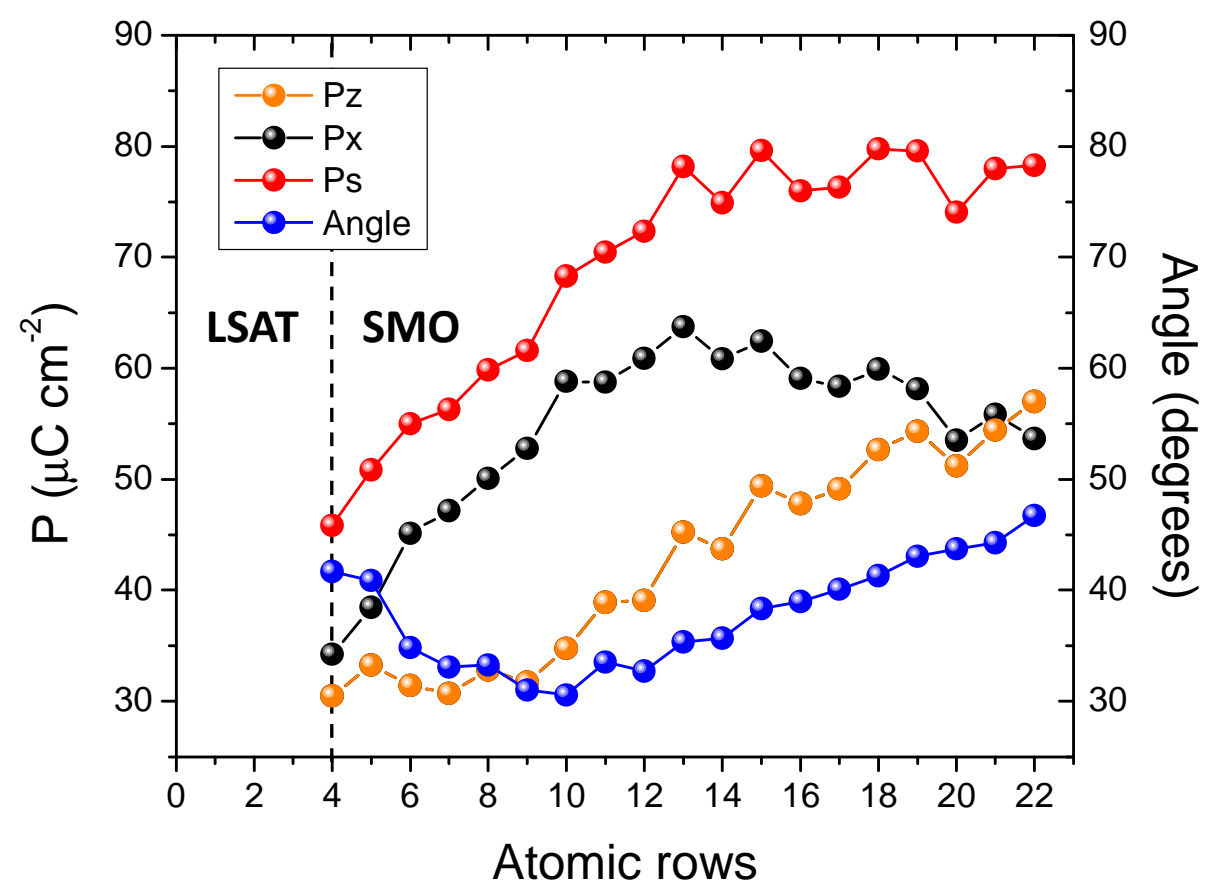

b

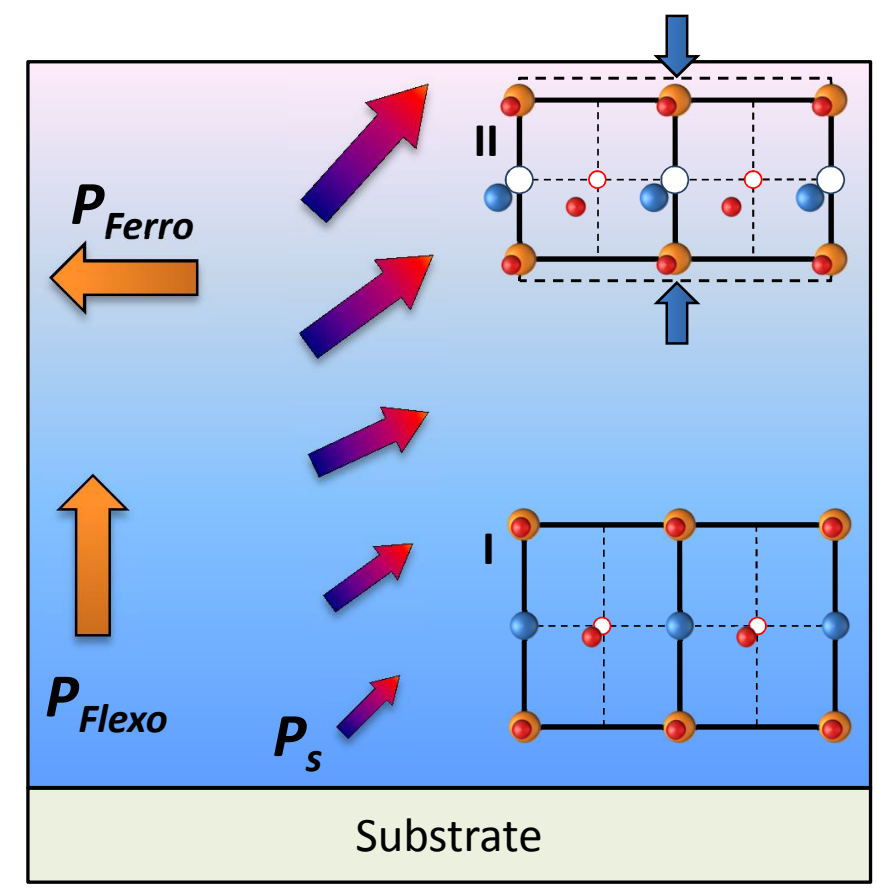


a

b
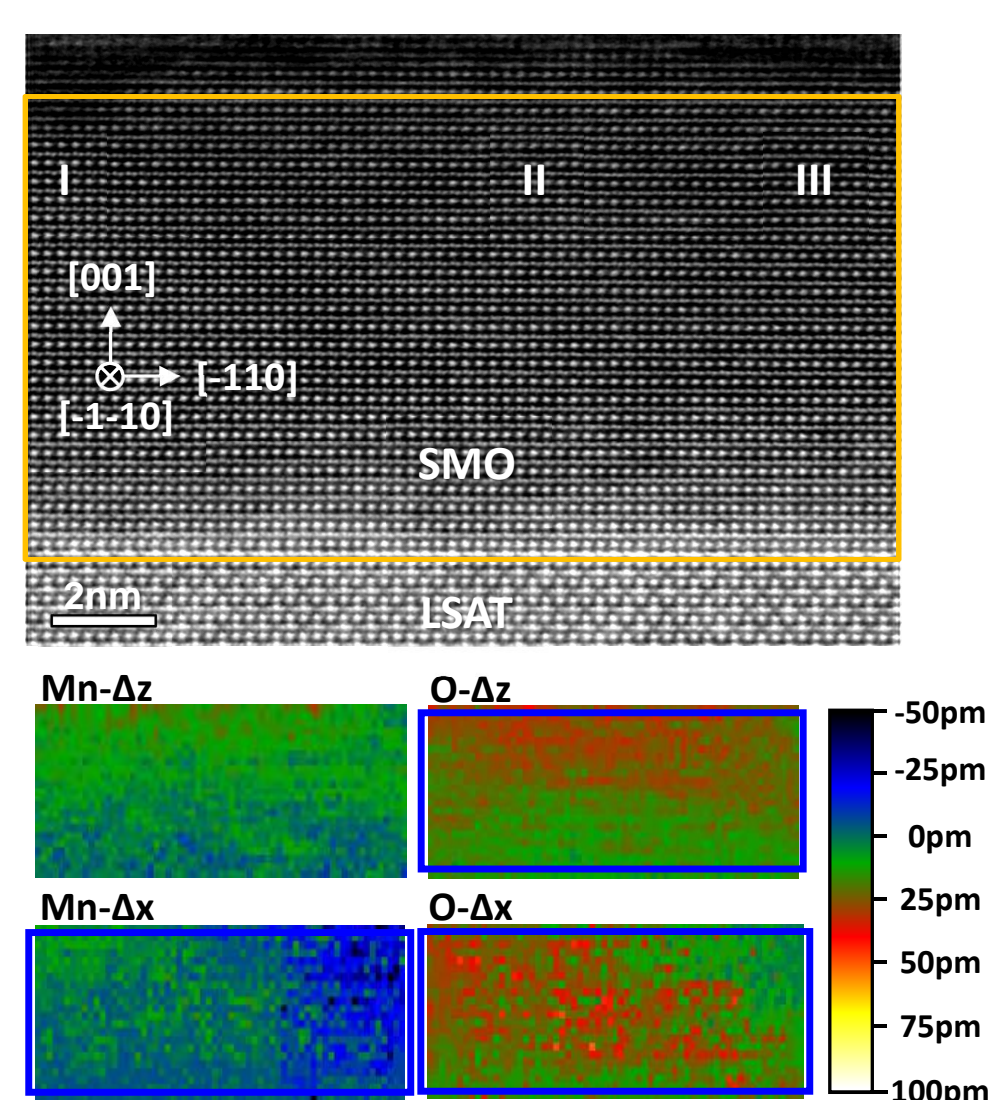

C

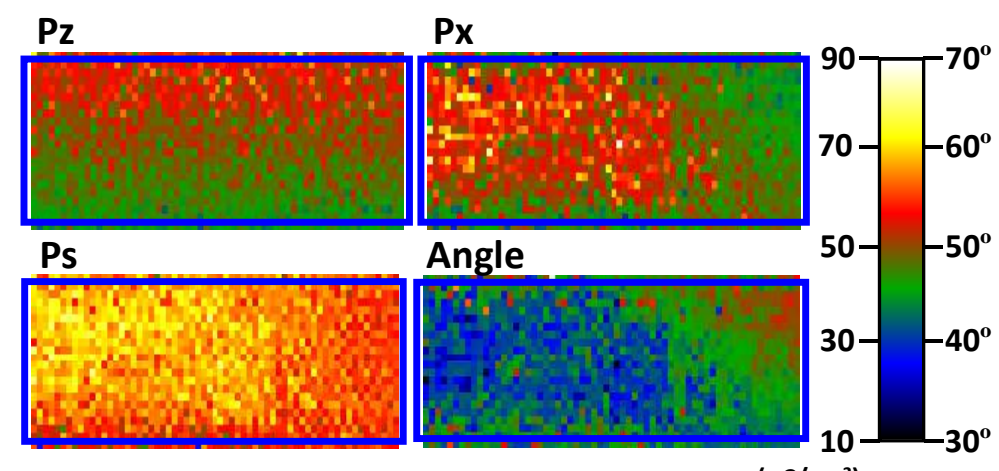

d

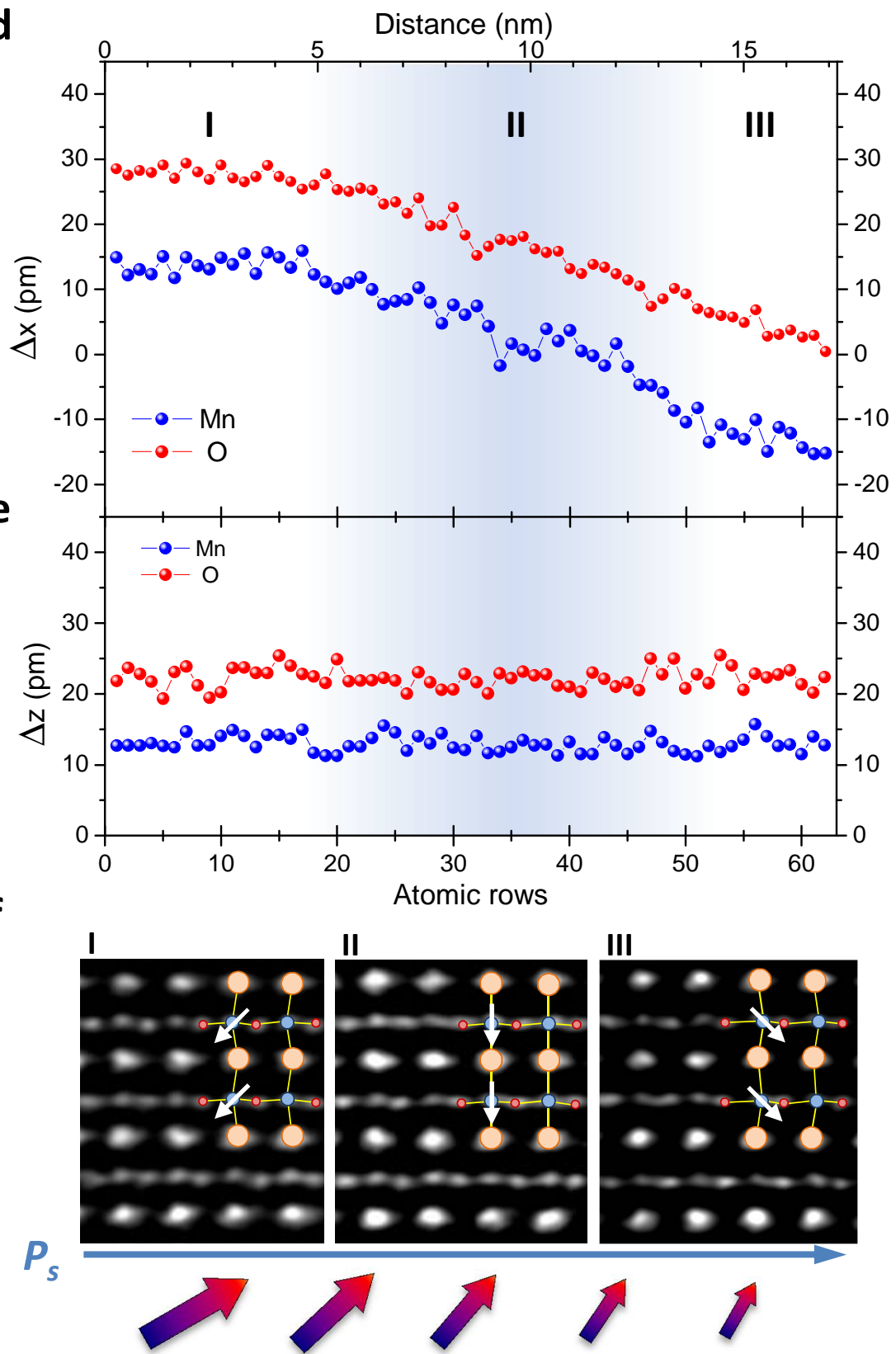

ACS Paragon Plus Environment 


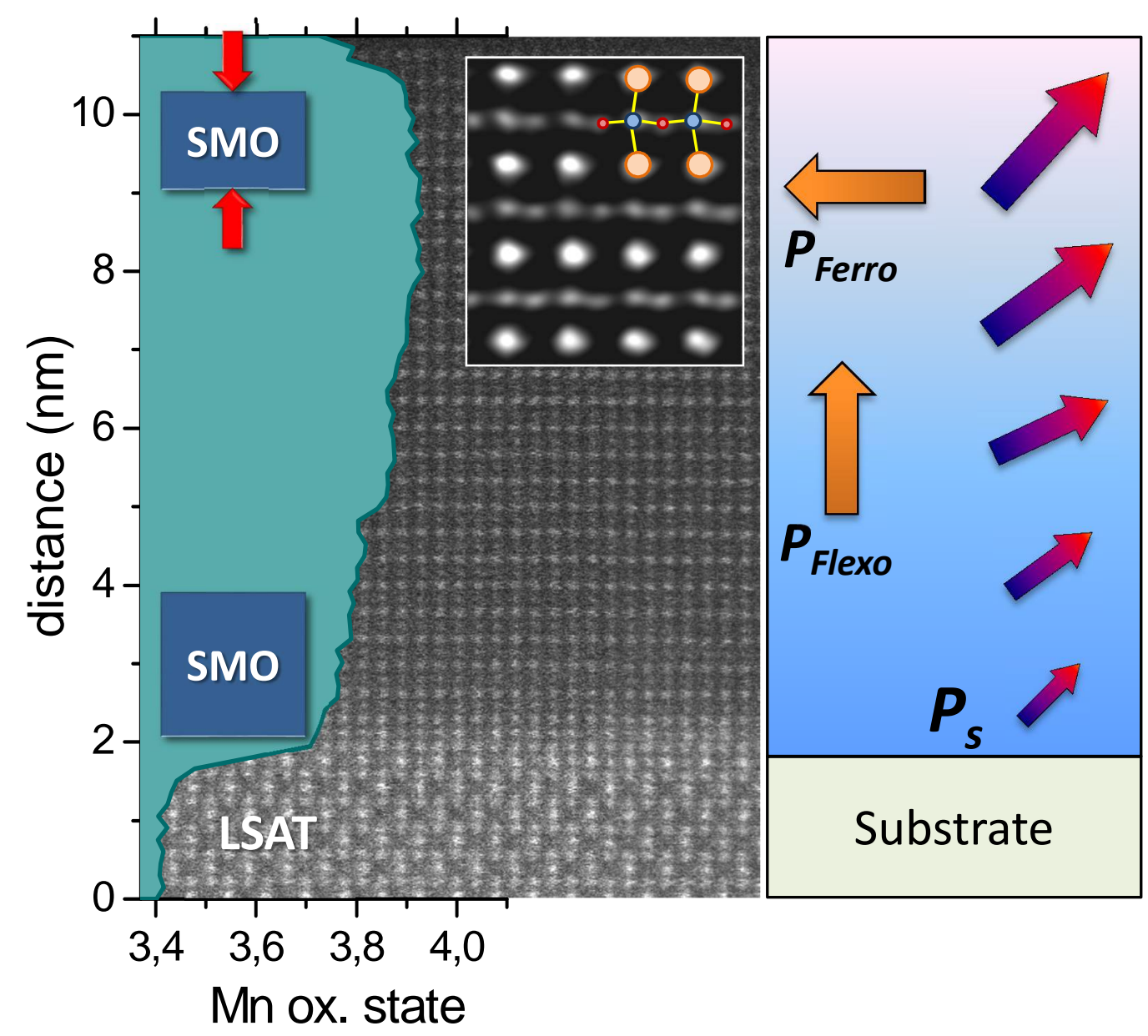

